\title{
Holomorphy in Pseudo-Fuclidean Spaces and the Classic Electromagnetic Theory
}

\author{
Vad L Negulesa \\ vlulune@googlemail.com
}

\begin{abstract}
A new concept of holomorphy in pseudo-Eudidean spaces is briefly presented. The set of extended Cauchy-Riemannn differential equations, which are verified by the holomorphic functions, is obtained. A form of the general pseudo-rotation matrix was developed. The generalized d'Alembert- operator and extended Poisson's equations are defined. Applying these results to the relativistic space-time, the charge conservation and general Maxwell equations are derived.
\end{abstract}

\section{Introduction}

In a paper [1], published in 1981, Salingaros proposed an extension of the Cauchy-Riemann equations of holomorphy to fields in higher-dimensional spaces. He formulated the theory of holomorphic fields by using Cifford algebras [2]. In the Minkowski space-time he found out that the equations of holomorphy are identical with the Maxwell equations in vacuum.

In the present article we introduce a different definition of monogenity/holomorphy applied to vector functions in a pseudo-Eudidean space. This enables us to obtain a set of equations, which applied to the Minkowski space-time, lead to general Maxwell equations and to the charge conservation law. All physical quantities involved in the ongoing presentation are expressed in geometric units [3], i.e. meters.

\section{Preliminary}

\subsection{Pseudo-rotation and its transformation matrix}

Let us consider a Riemannian n-dimensional space with the metric [4]:

$$
d^{2} s_{x}=\sum_{i, k=1}^{n} g_{i k} d x_{i} d x_{k}
$$

If the $g_{i k}$ coefficients are constant, then the space is called pseudo-Eudidean and the coordinate system is rectilinear. Using linear transformations we obtain a new coordinates system:

$$
x_{i}^{\prime}=x_{i}^{\prime}\left(x_{1}, x_{2}, \ldots \ldots \ldots . . . . x_{n}\right)
$$


Below we have the expression of the Jacobian matrix of this transformation.

$$
J=\left[\frac{\partial x^{\prime}}{\partial x_{1}}, \frac{\partial x^{\prime}}{\partial x_{2}}, \ldots \frac{\partial x^{\prime}}{\partial x_{n}}\right]=\left(\begin{array}{llll}
\frac{\partial x_{1}^{\prime}}{\partial x_{1}} & \frac{\partial x_{1}^{\prime}}{\partial x_{2}} & \cdot & \frac{\partial x_{1}^{\prime}}{\partial x_{n}} \\
\frac{\partial x_{2}^{\prime}}{\partial x_{1}} & \frac{\partial x_{2}^{\prime}}{\partial x_{2}} & \cdot \frac{\partial x_{2}^{\prime}}{\partial x_{n}} \\
\frac{\partial \dot{x}_{n}^{\prime}}{\partial x_{1}} & \frac{\partial \dot{x}_{n}^{\prime}}{\partial x_{2}} & \cdot & \frac{\partial \dot{x}_{n}^{\prime}}{\partial x_{n}}
\end{array}\right)
$$

If the value of $d s_{x}$ remains unmodified, then this transformation will be generally named a pseudorotation The transformation becomes pure rotation in the case of Eudidean spaces.

$$
\begin{aligned}
& d^{2} s_{x}=\sum_{i, k=1}^{n} g_{i k} d x_{i} d x_{k}=\sum_{i, k}^{n} g_{i k}^{\prime} d x_{i}^{\prime} d x_{k}^{\prime}=d^{2} s_{x^{\prime}} \\
& \frac{d^{2} s_{x^{\prime}}}{d^{2} s_{x}}=1
\end{aligned}
$$

We will consider further only transformations where $g_{i k}=g_{i k}^{\prime}$.

Developing the differentials in the right side of the first equation (1.4) and identifying, it obtains the following important relationship:

$$
g_{j p}=\sum_{i, k=1}^{n} g_{i k} \frac{\partial x_{i}^{\prime}}{\partial x_{j}} \frac{\partial x_{k}^{\prime}}{\partial x_{p}}
$$

\subsection{Holomorphy in n-dimensional spaces}

If it considered a vector field $\mathbf{f}=\left(f_{1}, f_{2}, \ldots . . f_{n}\right)$, defined on an $n$-dimensional space, then its Jacobi matrix is as follows:

$$
J=\left[\frac{\partial \mathrm{f}}{\partial x_{1}}, \frac{\partial \mathrm{f}}{\partial x_{2}}, \ldots \frac{\partial \mathrm{f}}{\partial x_{n}}\right]=\left(\begin{array}{llll}
\frac{\partial f_{1}}{\partial x_{1}} & \frac{\partial f_{1}}{\partial x_{2}} & \cdot \frac{\partial f_{1}}{\partial x_{n}} \\
\frac{\partial f_{2}}{\partial x_{1}} & \frac{\partial f_{2}}{\partial x_{2}} & \cdot \frac{\partial f_{2}}{\partial x_{n}} \\
\frac{\partial f_{n}}{\partial x_{1}} & \frac{\partial f_{n}}{\partial x_{2}} & \cdot & \frac{\partial f_{n}}{\partial x_{n}}
\end{array}\right)
$$

The differential of this field function can be written as:

$$
d \mathbf{f}=\left(d f_{1}, d f_{2}, \ldots . . d f_{n}\right)
$$


Using the metric definition (1.1) we may write the norm of this differential expression $d^{2} s_{f}=\sum_{i, k=1}^{n} g_{i k} d f_{i} d f_{k}$

\section{Definition}

A vector fieldf $=\left[\mathrm{f}_{1}(\mathrm{x}), \mathrm{f}_{2}(\mathrm{x}), \ldots \ldots \ldots \mathrm{f}_{\mathrm{n}}(\mathrm{x})\right]$, where $\mathrm{f}_{\mathrm{i}}(\mathrm{x})=\mathrm{f}_{\mathrm{i}}\left(\mathrm{x}_{1}, \mathrm{x}_{2}, \ldots . \mathrm{x}_{\mathrm{n}}\right)$, is said to be monogenic at point $x$ of the space if the ratio:

$$
\frac{\mathrm{d}^{2} \mathrm{~s}_{\mathrm{f}}}{\mathrm{d}^{2} \mathrm{~s}_{\mathrm{x}}}=\frac{\sum_{i, k=1}^{n} g_{i k} \mathrm{df}_{\mathrm{i}} \mathrm{df}_{\mathrm{k}}}{\sum_{i, k=1}^{n} g_{i k} d x_{i} \mathrm{dx}_{\mathrm{k}}}= \pm \Omega^{2}(x),
$$

exists and is unique at this point. If a vector field $f$ is monogenic in all the points belonging to a set $D$ in space, then $\mathrm{f}$ is holomorphic in the set $\mathrm{D}$.

For further developments we consider only the sign + in the right side of the equation (1.8). The uniqueness condition (1.8) requires that:

$$
g_{j p}=\sum_{i, k=1}^{n} g_{i k}\left(\frac{1}{\Omega} \frac{\partial f_{i}}{\partial x_{j}}\right)\left(\frac{1}{\Omega} \frac{\partial f_{k}}{\partial x_{p}}\right)
$$

Comparing with (1.5) and (1.3) it obtains the following set of equations:

$$
\frac{1}{\Omega} \frac{\partial f_{i}}{\partial x_{j}}=\frac{\partial x_{i}^{\prime}}{\partial x_{j}},
$$

where $i, j=1,2, \ldots \ldots \ldots . . . n$.

Equations (1.10) can be considered as the extension of the Caudhy-Riemam equations to an ndimensional space. Further it will be considered only pseudo-Eudidean spaces where:

$$
\begin{aligned}
& g_{i k}=g^{\prime}{ }_{k i}=0 \\
& g_{i i}=g^{\prime}{ }_{i i}=c_{i}
\end{aligned}
$$

More than that we may state, for simplicity, that ci is either 1 or -1 .

\subsection{Pseudo-rotation matrices and associated Cauchy-Riemann equations}

Let us consider a $n \times n$ matrix $\mathrm{M}$, which performs the coordinate transformation $\mathrm{X} \rightarrow \mathrm{X}^{\prime}$ in an $\mathrm{n}$ dimensional space: 


$$
M=\left(\begin{array}{cccc}
a_{11} & a_{12} & \cdot & a_{1 n} \\
a_{21} & a_{22} & \cdot & a_{2 n} \\
\cdot & \cdot & \cdot & \cdot \\
a_{n 1} & a_{n 2} & \cdot & a_{n n}
\end{array}\right)
$$

$M$ is a pseudo-rotation matrix if and only if its columns verify the equation (1.5). This is the necessary and sufficient condition for $\mathrm{M}$ to be a called a pseudo-rotation matrix.

a. As a first example we consider one of the rotation matrices in the two dimensional Eudidean space, where $c_{1}=c_{2}=1$.

$$
M=\left[\begin{array}{cc}
\cos \gamma & \sin \gamma \\
-\sin \gamma & \cos \gamma
\end{array}\right]
$$

Using (1.10) it obtains the original Cauchy-Riemann equations, known from complex analysis

$$
\begin{aligned}
& \frac{\partial f_{1}}{\partial x_{1}}=\frac{\partial f_{2}}{\partial x_{2}} \\
& \frac{\partial f_{1}}{\partial x_{2}}=-\frac{\partial f_{2}}{\partial x_{1}}
\end{aligned}
$$

Considering the definition (1.8), the conditions (1.12) are not unique. An alternate valid form of a rotation matrix in this space could be:

$$
M=\left[\begin{array}{cc}
\cos \gamma & \sin \gamma \\
\sin \gamma & -\cos \gamma
\end{array}\right]
$$

Consequently the extended Cauchy-Riemann equations look differently:

$$
\begin{aligned}
& \frac{\partial f_{1}}{\partial x_{1}}=-\frac{\partial f_{2}}{\partial x_{2}} \\
& \frac{\partial f_{1}}{\partial x_{2}}=\frac{\partial f_{2}}{\partial x_{1}}
\end{aligned}
$$

In both cases the functions are harmonic and satisfy the Laplace's equations:

$$
\begin{aligned}
& \frac{\partial^{2} f_{1}}{\partial x_{1}^{2}}+\frac{\partial^{2} f_{1}}{\partial x_{2}^{2}}=0 \\
& \frac{\partial^{2} f_{2}}{\partial x_{1}^{2}}+\frac{\partial^{2} f_{2}}{\partial x_{2}^{2}}=0
\end{aligned}
$$

b. A pseudo-rotation matrix in a two-dimensional pseudo-Eudidean space, where $c_{1}=1$ and $c_{2}=1$, can have the following form: 


$$
M=\left[\begin{array}{cc}
\cosh \gamma & -\sinh \gamma \\
-\sin \gamma & \cosh \gamma
\end{array}\right]
$$

$M$ is the matrix of the Lorenz transformations in the two dimensional $\mathbf{s p a c e}\left(\mathrm{x}_{2}\right)$-time $\left(\mathrm{x}_{1}\right)$, and the corresponding Cauchy-Riemann equations system is shown below:

$$
\begin{aligned}
& \frac{\partial f_{1}}{\partial x_{1}}=\frac{\partial f_{2}}{\partial x_{2}} \\
& \frac{\partial f_{1}}{\partial x_{2}}=\frac{\partial f_{2}}{\partial x_{1}}
\end{aligned}
$$

The above functions satisfy the wave equation in one space dimension.

$$
\begin{aligned}
& \frac{\partial^{2} f_{1}}{\partial x_{1}^{2}}=\frac{\partial^{2} f_{1}}{\partial x_{2}^{2}} \\
& \frac{\partial^{2} f_{2}}{\partial x_{1}^{2}}=\frac{\partial^{2} f_{2}}{\partial x_{2}^{2}}
\end{aligned}
$$

\subsection{Generalized d'Alembert operator and extended Poisson's equations}

As we have seen in the previous paragraph, the pseudo-rotation matrices on the same space are not identical and their freedom degree grows with the dimensions number, $\mathrm{n}$. This implies that for the same type of space there are different Cauchy-Riemann equations which provide necessary conditions for a vector field $\mathbf{f}$ to be holomorphic. One of possible forms of a general pseudo-rotation matrix may be as follows:

$$
M=\left(\begin{array}{cccc}
a_{11} & a_{12} & \cdot & a_{1 n} \\
a_{21} & a_{22} & \cdot & a_{2 n} \\
\cdot & \cdot & \cdot & \cdot \\
a_{n 1} & a_{n 2} & , & a_{n n}
\end{array}\right)=\left(\begin{array}{cccc}
\alpha_{1} & \alpha_{2} & \cdot & \alpha_{n} \\
\alpha_{2} & c_{2}-\frac{\alpha_{2}{ }^{2}}{c_{1}-\alpha_{1}} & \cdot & -\frac{\alpha_{2} \alpha_{n}}{c_{1}-\alpha_{1}} \\
\cdot & \cdot & \cdot & \cdot \\
\alpha_{n} & -\frac{\alpha_{2} \alpha_{3}}{c_{1}-\alpha_{1}} & \cdot & c_{n}-\frac{\alpha_{n}{ }^{2}}{c_{1}-\alpha_{1}}
\end{array}\right)
$$

It can be verified that all columns of M satisfy the equation (1.5), and also that:

$$
a_{i k}=a_{k i}
$$

Now let us process the elements of the diagonal which starts with $\alpha_{1}$, in according with the following relationship

$$
\begin{aligned}
& S_{\text {diagonal }}=\sum_{i=1}^{n} c_{i} a_{i i} \\
& S_{\text {diagonal }}=c_{1} \alpha_{1}+c_{2}\left(c_{2}-\frac{\alpha_{2}{ }^{2}}{c_{1}-\alpha_{1}}\right)+c_{3}\left(c_{3}-\frac{\alpha_{3}{ }^{2}}{c_{1}-\alpha_{1}}\right) \ldots \ldots .+c_{n}\left(c_{n}-\frac{\alpha_{n}{ }^{2}}{c_{1}-\alpha_{1}}\right) \\
& S_{\text {diagonal }}=n-1+\frac{\alpha_{1}-c_{1} \alpha_{1}{ }^{2}-c_{2} \alpha_{2}{ }^{2} \ldots \ldots .-c_{n} \alpha_{n}{ }^{2}}{c_{1}-1}=n-1+\frac{\alpha_{1}-c_{1}}{c_{1}-\alpha_{1}}=n-2
\end{aligned}
$$


Vlad L. Negulescu, "Holomorphy in Pseudo-Euclidean Spaces and the Classic Electromagnetic Theory", Transactions on Networks and Communications, Volume 7 No. 4, August (2019); pp: 27-36

For the four-dimensional Minkowski space-time, $c_{1}=1, c_{2}=c_{3}=c_{1}=1$, the corresponding matrix becomes:

$$
M=\left[\begin{array}{cccc}
\alpha_{1} & \alpha_{2} & \alpha_{3} & \alpha_{4} \\
\alpha_{2} & 1+\frac{\alpha_{2}{ }^{2}}{1+\alpha_{1}} & \frac{\alpha_{2} \alpha_{3}}{1+\alpha_{1}} & \frac{\alpha_{2} \alpha_{4}}{1+\alpha_{1}} \\
\alpha_{3} & \frac{\alpha_{3} \alpha_{2}}{1+\alpha_{1}} & 1+\frac{\alpha_{3}{ }^{2}}{1+\alpha_{1}} & \frac{\alpha_{3} \alpha_{4}}{1+\alpha_{1}} \\
\alpha_{4} & \frac{\alpha_{4} \alpha_{2}}{1+\alpha_{1}} & \frac{\alpha_{4} \alpha_{3}}{1+\alpha_{1}} & 1+\frac{\alpha_{4}{ }^{2}}{1+\alpha_{1}}
\end{array}\right]
$$

Taking the appropriate substitutions and computing, it obtains the matrix of the standard Lorenz transformations as you can see in the reference [5] (equations 1.17 and the corresponding matrix).

Using the equations (1.17), (1.18) and (1.10) it obtains the following relationships:

$$
\begin{aligned}
& \frac{\partial \mathrm{f}_{\mathrm{i}}}{\partial \mathrm{x}_{\mathrm{k}}}=\frac{\partial \mathrm{f}_{\mathrm{k}}}{\partial \mathrm{x}_{\mathrm{i}}} \\
& \sum_{\mathrm{i}=1}^{\mathrm{n}} \mathrm{c}_{\mathrm{i}} \frac{\partial \mathrm{f}_{\mathrm{i}}}{\partial \mathrm{x}_{\mathrm{i}}}=(\mathrm{n}-2) \Omega=\Lambda
\end{aligned}
$$

Processing (1.19) it arrives to the following expression:

$$
\sum_{i=1}^{n} c_{i} \frac{\partial^{2} f_{k}}{\partial x_{i}^{2}}=\partial^{2} f_{k}=\frac{\partial \Lambda}{\partial x_{k}}
$$

Further the symbol $\partial^{2}$ will be named d'Alembert operator of the n-dimensional pseudo-Eudidean space. The equation (1.20) is denominated the extended Poisson's equation in the same space.

For $n=2$ it obtains the Laplace equations and the wave equations, previously developed in the paragraph 1.2 .

For the Minkowski space-time, with the signature $(-1,1,1,1)$, it will be used further the standard denomination of the coordinates, i.e. $t, x_{1}, x_{2}, x_{3}$.

The corresponding vector field has the following expression:

$$
\mathrm{f}=\left(T, X_{1}, X_{2}, X_{3}\right)
$$

Using equations (1.20) it obtains:

$$
\begin{gathered}
\partial^{2} \mathrm{~T}=\frac{\partial \Lambda}{\partial \mathrm{t}} \\
\partial^{2} \mathrm{X}_{\mathrm{i}}=\frac{\partial \Lambda}{\partial \mathrm{x}_{\mathrm{i}}}
\end{gathered}
$$

The symbol $\Lambda=2 \Omega$ represents a function at the pointP $\left(\mathrm{t}, \mathrm{x}_{1}, \mathrm{x}_{2}, \mathrm{x}_{3}\right)$.

$\mathrm{X}_{1}, \mathrm{X}_{2}, \mathrm{X}_{3}$ are the components of the following vector in the three-dimensional Eudidean space: 


$$
\mathrm{X}=\sum_{i=1}^{3} X_{i} \mathrm{e}_{\mathrm{i}}
$$

where $e_{i}$ are unit vectors along the Cartesian axes of this space.

The last three equations of the system (1.22) will be packed together, and so the system takes the following format:

$$
\begin{aligned}
& \partial^{2} \mathbf{X}=\nabla \Lambda \\
& \partial^{2} \mathrm{~T}=\frac{\partial \Lambda}{\partial \mathrm{t}}
\end{aligned}
$$

In the systemabove it was used the "del" operator in the three dimensional Euclidean space.

If we consider the pair of inhomogeneous wave equations ${ }^{6}$ for electromagnetic potentials, then the system (1.24) shows a perfect similarity. We are very tempted to identify $T$ with the electro-magnetic scalar potential and the vector $\mathbf{X}$ with the vector potential, but it does not work because the first equation of the system (1.19) requires that the curl-operator or rotation-operator of X must be zero. This is not generally valid for a real electro-magnetic vector-potential.

\section{Cassical Electrodynamics and Maxwell equations}

\subsection{Alternative Cauchy-Riemann equations in Space-Time.}

There is a class of matrices in the Minkowski space-time which fulfills the following relations:

$$
\begin{aligned}
& \sum_{\mathrm{i}=1}^{4} \mathrm{a}_{\mathrm{ii}}=0 \\
& \mathrm{a}_{12}+\mathrm{a}_{21}=\mathrm{c}_{2} \\
& \mathrm{a}_{13}+\mathrm{a}_{31}=\mathrm{c}_{3} \\
& \mathrm{a}_{14}+\mathrm{a}_{41}=\mathrm{c}_{4}
\end{aligned}
$$

Replacing by partial derivatives, in according with equations (1.10) and using the usual coordinate's notation for Minkowski space-time, it obtains:

$$
\begin{aligned}
& \frac{\partial \mathrm{T}}{\partial \mathrm{t}}+\nabla \bullet \mathrm{X}=0 \\
& \nabla \mathrm{T}+\frac{\partial \mathrm{X}}{\partial \mathrm{t}}=\frac{\partial \mathrm{C}}{\partial \mathrm{t}}
\end{aligned}
$$

We also can obtain the equations system (2.2) considering a vector field, $f=\left(T, X_{1}^{\prime}, X_{2}^{\prime}, X_{3}^{\prime}\right)$ in Minkowski space-time which fulfils the conditions (1.19). If $X$ ' represents the corresponding vector in the three dimensional Eudidean space, then respective equations become: 


$$
\begin{aligned}
& -\frac{\partial \mathrm{T}}{\partial \mathrm{t}}+\nabla \bullet \mathrm{X}^{\prime}=\Lambda=\nabla \bullet \mathrm{C} \\
& \nabla \mathrm{T}-\frac{\partial \mathrm{X}^{\prime}}{\partial \mathrm{t}}=0
\end{aligned}
$$

Now let us make the substitution: $\mathrm{X}=\mathrm{C}-\mathrm{X}^{\prime}$

Replacing in (2.3) we arrive again at the system (2.2). The second equation of the system (2.2) implies that:

$$
\nabla \times \mathrm{X}=\nabla \times \mathrm{C}
$$

Now let us convert to the SI system (see reference [3]) identifying the scalar part with the scalar potential and the vector part with the vector potential. Further we will use the symbols shown in the reference [6] for these potentials. It obtains the following system of equations:

$$
\begin{aligned}
& \frac{\partial \Phi}{\partial \mathrm{t}}+\mathrm{c}^{2} \nabla \mathrm{A}=0 \\
& \nabla \Phi+\frac{\partial \mathrm{A}}{\partial \mathrm{t}}=\frac{\partial \mathrm{C}}{\partial \mathrm{t}}
\end{aligned}
$$

Where c is the velocity of light in SI units system. Processing further we get:

$$
\begin{aligned}
& \nabla^{2} \Phi-\frac{1}{\mathrm{c}^{2}} \frac{\partial^{2} \Phi}{\partial \mathrm{t}^{2}}=\nabla \bullet\left(\frac{\partial \mathrm{C}}{\partial \mathrm{t}}\right) \\
& \nabla(\nabla \bullet \mathrm{A})-\frac{1}{\mathrm{c}^{2}} \frac{\partial^{2} \mathrm{~A}}{\partial \mathrm{t}^{2}}=-\frac{1}{\mathrm{c}^{2}} \frac{\partial^{2} \mathrm{C}}{\partial \mathrm{t}^{2}}
\end{aligned}
$$

But:

$\nabla(\nabla \bullet \mathrm{A})=\nabla^{2} \mathrm{~A}+\nabla \times(\nabla \times \mathrm{A})$, and finally the system (2.6) becomes:

$$
\begin{aligned}
& \nabla^{2} \mathrm{~A}-\frac{1}{\mathrm{c}^{2}} \frac{\partial^{2} \mathrm{~A}}{\partial \mathrm{t}^{2}}=-\frac{1}{\mathrm{c}^{2}} \frac{\partial^{2} \mathrm{C}}{\partial \mathrm{t}^{2}}-\nabla \times(\nabla \times \mathrm{A})=-\frac{1}{\mathrm{c}^{2}} \frac{\partial^{2} \mathrm{C}}{\partial \mathrm{t}^{2}}-\nabla \times(\nabla \times \mathrm{C}) \\
& \nabla^{2} \Phi-\frac{1}{\mathrm{c}^{2}} \frac{\partial^{2} \Phi}{\partial \mathrm{t}^{2}}=\nabla \bullet\left(\frac{\partial \mathrm{C}}{\partial \mathrm{t}}\right)
\end{aligned}
$$

\subsection{Charge oonservation and Maxwell's Equations}

Identifying (2.7) with inhomogeneous wave equations presented by Feynman [6] we find the expressions for charge and current density.

$$
\begin{aligned}
& \rho=-\varepsilon_{0} \nabla \bullet\left(\frac{\partial \mathrm{C}}{\partial \mathrm{t}}\right) \\
& \mathrm{j}=\varepsilon_{0}\left[\frac{\partial^{2} \mathrm{C}}{\partial \mathrm{t}^{2}}+\mathrm{c}^{2} \nabla \times(\nabla \times \mathrm{C})\right]
\end{aligned}
$$

Processing the equations (2.8) it obtains immediately the well known equation of chargeconsenvation: 


$$
\nabla \bullet \mathrm{j}=-\frac{\partial \rho}{\partial t}
$$

Using the equations (2.5 wecan write further the expressions of electric field intensity $\boldsymbol{E}$ and the magnetic induction $\mathbf{B}$ :

$$
\begin{aligned}
& \boldsymbol{E}=-\nabla \Phi-\frac{\partial \mathrm{A}}{\partial t}=-\frac{\partial \mathrm{C}}{\partial t}, \\
& \boldsymbol{B}=\nabla \times \mathrm{A}=\nabla \times \mathrm{C}
\end{aligned}
$$

\section{a. The first Maxwell'slaw}

Taking the divergence of $\mathbf{E}$ and using the first equation of the system (2.8) it obtains the first Maxwell's law:

$$
\nabla \bullet E=\frac{\rho}{\varepsilon_{0}}
$$

b. Thesecond Maxwell'slaw

Taking the curl of Eand comparing with the expression of $\mathbf{B}$ we get immediately the second law:

$$
\nabla \times \mathrm{E}=-\frac{\partial \mathrm{B}}{\partial t}
$$

\section{c. Thethird Maxwell'slaw}

The third Maxwell's equation is evident because $\mathbf{B}$ is curl $\mathbf{C}$ :

$$
\nabla \bullet \mathrm{B}=0
$$

\section{d. The fourth Maxwell'slaw}

Processing the second equation of the system (2.8) and taking into consideration (2.10) we found finally the fourth law:

$$
c^{2} \nabla \times \mathrm{B}=\frac{\mathbf{j}}{\varepsilon_{0}}+\frac{\partial \mathrm{E}}{\partial t}
$$

\section{Conclusion}

The Lorenz Transformation equations have been initially derived searching for a transformation, which leaves the Maxwell's equations invariant [7]. The immediate consequence of the Lorenztransformations is Enstein's Special Relativity. In the present contribution we found that any four-vector, which is holomorphic in a domain of the space-time, must verify the system (1.10). As a first application we rediscovered the law of charge conservation and all four Maxwell's equations.

\section{RUERANCE}

[1]. N. Salingaros, Eectromagnetismand the holomorphic properties of spacetime, J.Math.Phys. 22, 19191925 (1981).

[2]. K.Guerlebeck and W. Sproessig, Quaternionic and Cifford calculus for physicists and engineers (Gichester, Wiley, 1997). 
Vlad L. Negulescu, "Holomorphy in Pseudo-Euclidean Spaces and the Classic Electromagnetic Theory", Transactions on Networks and Communications, Volume 7 No. 4, August (2019); pp: 27-36

[3]. Geometrized Units System, http://en.wikipedia.org/wiki/Geometrized_unit_system (accessed May 25, 2019)

[4]. R. Adler, M. Bazin, M. Schiffer, Introduction to general relativity (New York, McGraw-Hill, 1965)

[5]. Vlad L Negulescu, Motion analysis of partides using the hyper-complex numbers representation, Open Access Journal of Mathematical and Theoretical Physics, Volume 2, Issue 1, 2019, https://medcraveonline.com/OAJMTP/OAJMTP-02-00047.pdf

[6]. R.P. Feynman, R.B. Leighton, M. Sands, The Feynman lectures on physics, 2. Mainly electromagnetism and matter (Reading, Mass., Addison-Wesley, 1969).

[7]. Oleg D. Jefimenko, On the Relativistic Invariance of Maxwell's Equations, Z Naturforsch. 54a, 637644 (1999, http:// đn.mpdl.mpg.de/data/Reihe A/54/ZNA-1999-54a-0637.pdf 